\title{
Fases identificables en la zona de interacción de placas combustibles dispersas $\mathrm{U}_{3} \mathrm{Si}_{2} / \mathrm{Al}$
}

\section{Phases identified in the interaction zone of $\mathrm{U}_{3} \mathrm{Si}_{2} / \mathrm{Al}$ dispersion fuel plates}

\author{
Marcela Inés Mirandou ${ }^{1}$, Ricardo Martín Sanabria ${ }^{1}$, Silvia Noemí Balart ${ }^{2}$ \\ Sergio FabiánAricó ${ }^{2,3}$, Jorge Omar Fabro ${ }^{4}$
}

\author{
${ }^{1}$ Departamento Estructura y Comportamiento - Gerencia Materiales - Gerencia de Área Energía Nuclear (GAEN) - \\ Comisión Nacional de Energía Atómica (CNEA) - Av. Gral. Paz 1499, B1650KNA, San Martín, Buenos Aires, Argenti- \\ na \\ e-mail: mirandou@cnea.gov.ar; ricardosanabria@cnea.gov.ar \\ ${ }^{2}$ Departamento Transformaciones y Propiedades - Gerencia Materiales - GAEN - CNEA, Buenos Aires, Buenos Aires, \\ Argentina. \\ e-mail: balart@ cnea.gov.ar \\ ${ }^{3}$ Instituto de Tecnología "Jorge Sábato" - UNSAM/CNEA, San Martín, Buenos Aires, Argentina. \\ e-mail: arico@cnea.gov.ar \\ ${ }^{4}$ Departamento Elementos Combustibles para Reactores de Investigación (ECRI) - Gerencia de Ciclo del Combustible \\ Nuclear-CNEA, Buenos Aires, Buenos Aires, Argentina. \\ e-mail: fabro@ cnea.gov.ar
}

\section{RESUMEN}

Los elementos combustibles dispersos fabricados con partículas de $\mathrm{U}_{3} \mathrm{Si}_{2}$ inmersas en una matriz de $\mathrm{Al}$ puro han mostrado a lo largo de los años un comportamiento muy estable bajo irradiación, en particular, en una configuración conocida como tipo placa. Estos combustibles han sido, o están siendo, usados para disminuir el enriquecimiento del U del núcleo de un gran número de reactores de investigación en todo el mundo sin que esto comprometa su rendimiento. En este sentido, resulta lícito extender este buen comportamiento a los compuestos formables por interdifusión entre el siliciuro y el Al durante la fabricación.

Por otro lado, en estos últimos años se ha invertido un gran esfuerzo en calificar un combustible de mayor densidad como lo son las aleaciones U(Mo). Con este objetivo, placas o miniplacas con núcleos formados por partículas de $\mathrm{U}(\mathrm{Mo})$ dispersas en una matriz de $\mathrm{Al}(\mathrm{Si})$ están siendo estudiadas internacionalmente. Los estudios post-irradiación realizados sobre placas que atravesaron satisfactoriamente los ensayos de irradiación, junto con placas de referencia analizadas luego del proceso de fabricación, mostraron que este buen comportamiento está asociado a la formación (durante la fabricación) de una zona de interacción formada por fases ricas en Si ubicada rodeando a las partículas de U(Mo).

En este trabajo, dos placas fabricadas con partículas $\mathrm{U}_{3} \mathrm{Si}_{2}$ dispersadas en una matriz de $\mathrm{Al}$ puro fueron estudiadas luego del proceso de fabricación mediante microscopías óptica y electrónica de barrido, microanálisis dispersivo en longitud de onda y difracción de rayos X. Los resultados muestran la formación del compuesto $\mathrm{U}(\mathrm{Al}, \mathrm{Si})_{3}$ junto con otra fase de igual estructura cristalina que el compuesto $\mathrm{U}_{3} \mathrm{Si}_{2}$ pero con diferente volumen de celda. Esta información resultaría importante para la calificación del U(Mo) a la hora de seleccionar la matriz $\mathrm{Al}(\mathrm{Si})$ que promueva la formación de lo que podría considerarse "una zona de interacción adecuada".

Palabras clave: Combustibles nucleares, uranio, identificación de fases.

\section{ABSTRACT}

Fuel elements based on $\mathrm{U}_{3} \mathrm{Si}_{2}$ particles dispersed in a pure $\mathrm{Al}$ matrix (known as dispersion fuel element) are known to be very stable under irradiation, in plate type configurations. For this reason, they were or are being used to convert to low enriched uranium a large number of research reactors without significant loss in performance. According to this statement it is perfectly lawful to extend this good irradiation performance to 
any compound grown by interdiffusion between silicide and $\mathrm{Al}$ during the fabrication process.

On the other hand, a great effort is being carried out to qualify U(Mo) alloys as higher density fuel. In this sense, plates or miniplates with meats formed by $\mathrm{U}(\mathrm{Mo})$ particles dispersed in an $\mathrm{Al}(\mathrm{Si})$ matrix are being internationally studied. Post-irradiation examinations performed on plates that underwent satisfactorily irradiation tests were studied taking into account the analysis of plates after fabrication process. As a result it was observed that good irradiation behavior is associated to the formation (during fabrication) of an interaction layer formed by Si-containing phases which surrounds $\mathrm{U}(\mathrm{Mo})$ particles.

In this work two plates fabricated with $\mathrm{U}_{3} \mathrm{Si}_{2}$ particles dispersed in an $\mathrm{Al}$ matrix were analyzed by optical and scanning electron microscopies, wave length dispersive microanalysis and $\mathrm{x}$-ray diffraction after fabrication process. The results showed the formation of two phases: $\mathrm{U}(\mathrm{Al}, \mathrm{Si})_{3}$ and $\mathrm{U}_{3} \mathrm{Si}_{2}$ with modified cell volume. This information would be considered useful, in the $\mathrm{U}(\mathrm{Mo})$ qualification process, in order to select $\mathrm{Al}(\mathrm{Si})$ matrix that promotes the formation of what can considered "a proper interaction layer".

Keywords: Nuclear fuels, uranium, phase characterization.

\section{INTRODUCCIÓN}

La utilización de $\mathrm{U}$ de bajo enriquecimiento en aleaciones $\mathrm{U}(\mathrm{Mo})$ en fase $\gamma \mathrm{U}$ se está estudiando para su empleo como combustible nuclear tipo disperso o monolítico para la conversión de los reactores de investigación y producción de radioisótopos de alto flujo neutrónico [1,2]. En esta aleación, la adición de 7 a $10 \%$ en peso de Mo se utiliza para mantener, en condición metaestable a temperatura ambiente, la fase $\gamma \mathrm{U}$ que presenta la mejor respuesta bajo irradiación [1-3].

Un núcleo compuesto por partículas de U(Mo) dispersas en una matriz $\mathrm{Al}-\mathrm{Si}$ es una de las configuraciones más prometedoras para cumplir con la calificación de este combustible, en configuración dispersa [4], ya que se han obtenido resultados satisfactorios en algunas experiencias de irradiación [5]. Los ensayos post-irradiación junto con el análisis de las correspondientes placas sin irradiar, mostraron que el buen comportamiento se correlaciona con la formación, durante la fabricación, de una "zona de interacción (ZI) apropiada" alrededor de las partículas U(Mo) [6,7]. Sin embargo la calificación del U(Mo) todavía está en curso porque los recientes intentos de someter este combustible a niveles de alto quemado a potencias elevadas no han resultado totalmente exitosos [8-10].

En CNEA-Argentina se está estudiando cómo obtener las mejores características de la ZI (uniformidad, composición química, fases formables, etc.) con las menores modificaciones en el proceso de fabricación (actualmente utilizado para elementos combustibles de siliciuro) y la menor concentración global de Si en la matriz. Los resultados de la caracterización de los núcleos de miniplacas fabricadas con U $7 \%$ peso Mo atomizado y disperso en matrices $\mathrm{Al}-2 \%$ peso $\mathrm{Si}$ y $\mathrm{Al}-4 \%$ peso $\mathrm{Si}$ fueron presentados en diversas reuniones científicas [11,12]. En ambos trabajos se mostró cómo una concentración de $4 \%$ en peso de Si en la matriz base $\mathrm{Al}$ resulta eficiente para asegurar la disponibilidad de Si para formar una ZI delgada y uniforme que recubra por completo las partículas $\mathrm{U}(\mathrm{Mo})$ en miniplacas soldadas perimetralmente por completo. Esta ZI está formada exclusivamente por fases que contienen Si.

Por otra parte, es bien sabido que los elementos combustibles basados en partículas $\mathrm{U}_{3} \mathrm{Si}_{2}$ dispersas en una matriz de Al puro son estables bajo irradiación en configuraciones del tipo placa y se utilizan para reducir el enriquecimiento en un gran número de reactores de investigación sin pérdida significativa en el rendimiento. De este resultado se puede inferir que las fases que forman la ZI crecida durante el proceso de fabricación en este tipo de elementos combustibles también tienen un buen rendimiento bajo irradiación y seguramente pueden considerarse como "una zona de interacción apropiada."

De lo expuesto en párrafos anteriores se deduce la importancia de caracterizar este tipo de combustibles nucleares en su etapa previa a la irradiación por dos motivos diferentes. En primer lugar, para realizar una comparación con los resultados obtenidos en las miniplacas fabricadas con la aleación combustible U(Mo) [11,12]. En segundo lugar, para planificar futuros estudios en pares de difusión $\mathrm{U}(\mathrm{Mo}) / \mathrm{Al}(\mathrm{Si})$ tendientes a encontrar cuál es la concentración de $\mathrm{Si}$ en el componente $\mathrm{Al}(\mathrm{Si})$ que promueve la formación de una ZI lo más parecida posible a la identificada para los siliciuros.

En este trabajo se analizaron dos placas fabricadas con partículas $\mathrm{U}_{3} \mathrm{Si}_{2}$ dispersadas en una matriz de Al para obtener información acerca de la ZI que se forma durante el proceso de fabricación. Estas dos placas se diferencian entre sí por el tipo de enfriamiento final al cual fueron sometidas y son parte del material de control de dos núcleos combustibles actualmente en uso en dos reactores nucleares de investigación (uno de origen nacional y otro internacional). 


\section{MATERIALES Y MÉTODOS}

Las dos placas combustibles se fabricaron utilizando la metodología marco-tapa la cual incluye las siguientes etapas: fabricación de las partículas portadoras de $\mathrm{U}$ (siliciuro- $\left.\mathrm{U}_{3} \mathrm{Si}_{2}\right)$, fabricación del compacto $\left(\mathrm{U}_{3} \mathrm{Si}_{2}+\mathrm{Al}\right.$ ), montaje del compacto en un marco de AA6061 y colocación de las tapas de AA6061, soldadura TIG perimetral del conjunto con esquinas abiertas, laminación en caliente $\left(500^{\circ} \mathrm{C}\right)$ y un tratamiento térmico final $\left(1\right.$ hora a $\left.480^{\circ} \mathrm{C}\right)$ + laminado en frío. La única diferencia en el proceso de fabricación entre ambas placas es el enfriamiento al finalizar el tratamiento térmico final. Una de ellas fue enfriada en horno (placa denominada $\left.\mathrm{U}_{3} \mathrm{Si}_{2} / \mathrm{Al}-\mathrm{H}\right)$ mientras que la otra fue enfriada en aire $\left(\mathrm{U}_{3} \mathrm{Si}_{2} / \mathrm{Al}-\mathrm{A}\right)$.

El compuesto $\mathrm{U}_{3} \mathrm{Si}_{2}$ utilizado en la fabricación de cada núcleo proviene de diferentes partidas. Ambas se fabricaron en la CNEA por fusión en un horno de inducción utilizando uranio de bajo enriquecimiento $\left(20 \%{ }^{235} \mathrm{U}\right)$ y Si puro. Después de la fundición, el material se molió, utilizando un molino de bolas, y se tamizó para obtener el polvo. Las partículas de $\mathrm{U}_{3} \mathrm{Si}_{2}$ se mezclaron con $\mathrm{Al}$ puro y prensaron en frío para obtener compactos de densidad $4,8 \mathrm{gU} / \mathrm{cm}^{3}$.

Al finalizar el proceso de fabricación se cortaron muestras de cada placa. La preparación superficial para caracterizar el núcleo se inició con un desbaste mecánico grueso con el objetivo de remover una de las tapas de AA6061 y alcanzar el núcleo, luego se realizó un desbaste fino con papeles esmeriles de carburo de silicio de granulometría decreciente finalizando en 1200. El pulido mecánico se realizó con pasta de diamante hasta $1 \mu \mathrm{m}$.

La caracterización se realizó mediante las técnicas de microscopía óptica (MO-Olympus - BX60M), microscopía electrónica de barrido (SEM -Philips SEM 515 y FEI QUANTA 200), espectroscopía dispersiva en energía (EDS-EDAX Phoenix 3.2), espectroscopía dispersiva en longitud de onda (EPMA-WDS CAMECA SX Five) utilizando patrones de U, Si y Al de alta pureza y difracción de rayos X (XRDPANalytical-Empyrean con radiación $\mathrm{Cu}-\mathrm{K} \alpha$ ). Los difractogramas fueron analizados con el programa PowderCell 2.4 [13] el cual permite identificar las estructuras cristalinas de las fases presentes y estimar parámetros de red por comparación con difractogramas teóricos de las fases identificadas.

\section{RESULTADOS}

Al finalizar el proceso de fabricación de cada partida de polvo se realizó una identificación de fases presentes junto con determinaciones de concentración. La Figura 1 muestra que la única fase identificada en el polvo utilizado para la fabricación de ambas placas es $\mathrm{U}_{3} \mathrm{Si}_{2}$.

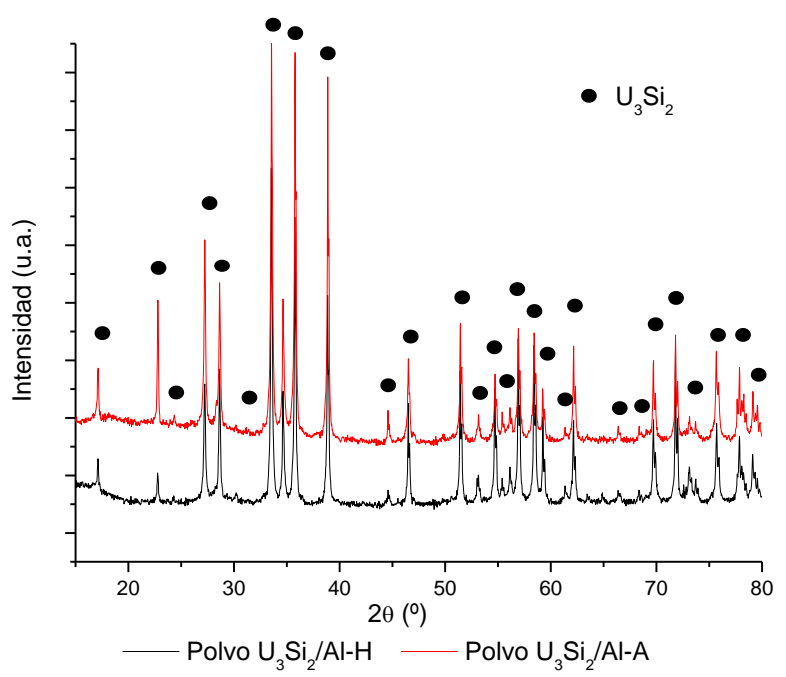

Figura 1: Identificación de fases al finalizar el proceso de fundición de las partículas portadoras de U.

Para obtener los valores de concentración de $\mathrm{U}$ y Si, se realizaron barridos lineales formados por 20 a 40 determinaciones puntuales con un paso de $3 \mu \mathrm{m}$ aproximadamente. Las partículas fueron elegidas al azar teniendo la precaución de evitar las fisuras y superficies de fractura características de materiales frágiles como el siliciuro de U utilizado en este trabajo. En la Tabla 1 se muestran los valores promedio obtenidos 
junto con su correspondiente dispersión. Estos resultados muestran una gran homogeneidad en el polvo obtenido pero con un valor promedio levemente más rico en $\mathrm{Si}$, por consiguiente, empobrecido en U respecto del valor estequiométrico (60\%at $\mathrm{U}$ y $40 \%$ at $\mathrm{Si}$ ) correspondiente a $\mathrm{U}_{3} \mathrm{Si}_{2}$.

Tabla 1: Concentración de U y Si en las partículas de $\mathrm{U}_{3} \mathrm{Si}_{2}$ obtenidas por fundición en horno de inducción. WDS.

\begin{tabular}{l|l}
\hline ELEMENTO & (\%AT) \\
\hline $\mathrm{Si}$ & $43.0 \pm 0.5$ \\
\hline $\mathrm{U}$ & $57.0 \pm 0.5$ \\
\hline
\end{tabular}

Al finalizar el proceso de fabricación ambas placas presentan una distribución homogénea de partículas de $\mathrm{U}_{3} \mathrm{Si}_{2}$ dentro del núcleo. La dispersión en el tamaño de partícula, una importante densidad de fisuras en las partículas y la porosidad observada al finalizar la preparación metalográfica surgen como consecuencia del proceso de laminación aplicado a un compuesto frágil como lo es el $\mathrm{U}_{3} \mathrm{Si}_{2}$. A modo de ejemplo se presenta la Figura 2 que permite observar el aspecto general del núcleo en la muestra $\mathrm{U}_{3} \mathrm{Si}_{2} / \mathrm{Al}-\mathrm{H}$.

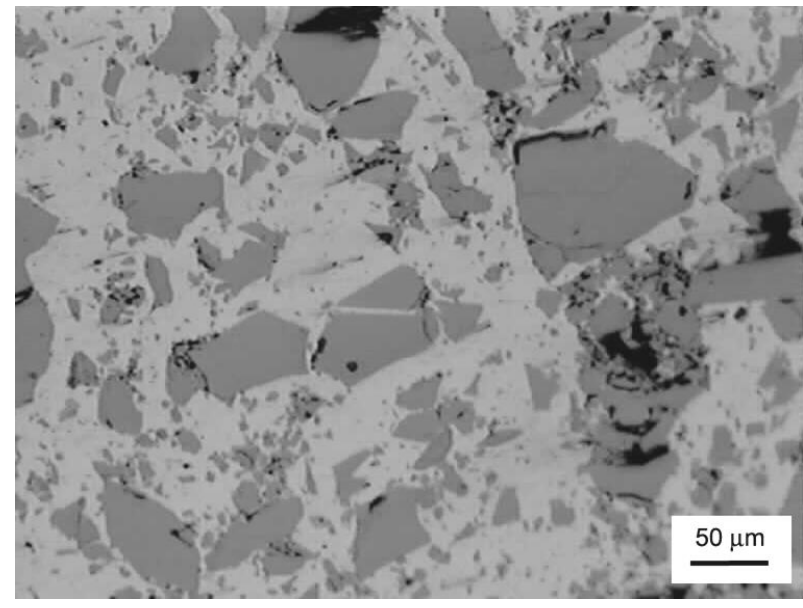

Figura 2: Muestra $\mathrm{U}_{3} \mathrm{Si}_{2} / \mathrm{Al}-\mathrm{H}$ : Aspecto general del núcleo al finalizar el proceso de fabricación.

Además, en el interior de algunas de las partículas de ambas placas combustibles se observaron inclusiones de $\sim 5 \mu \mathrm{m}$ de diámetro, como las que se muestran en la Figura 3, las cuales contienen $\mathrm{C}, \mathrm{O}$ y $\mathrm{N}$.

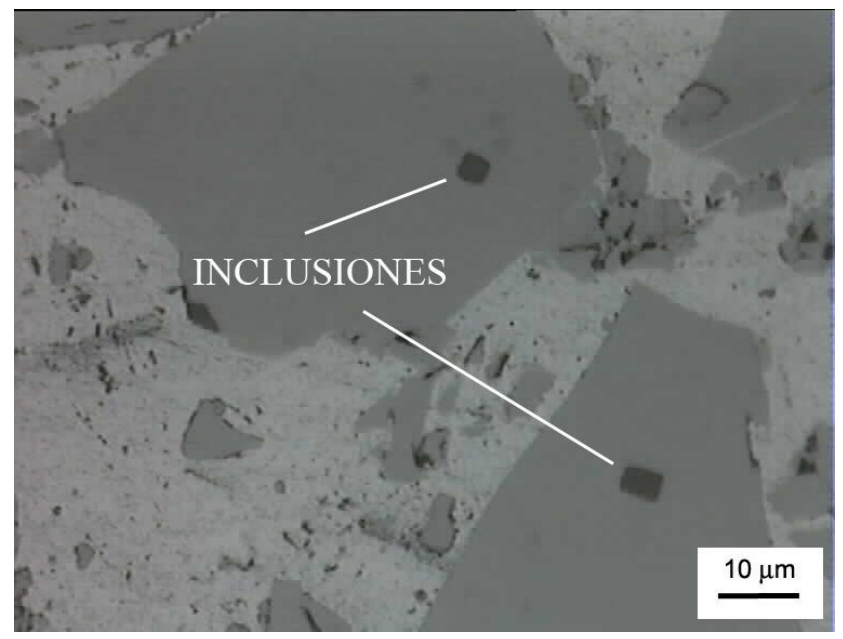

Figura 3: Muestra $\mathrm{U}_{3} \mathrm{Si}_{2} / \mathrm{Al}-\mathrm{A}$. Inclusiones en las partículas de $\mathrm{U}_{3} \mathrm{Si}_{2}$.

En la Figura 4 se presenta la identificación de fases que forman los núcleos de ambas placas una vez finalizado el proceso de fabricación. Ambas muestras presentan las mismas fases. En consecuencia, las diferentes condiciones de enfriamiento al finalizar el tratamiento térmico final no generan cambios 
significativos en cuanto a las fases identificables en el núcleo de una placa y, en consecuencia, en las fases que se forman en la ZI durante el proceso de fabricación.

Las fases $\mathrm{U}_{3} \mathrm{Si}_{2}$ y $\mathrm{Al}$ corresponden a los componentes que originalmente formaban el compacto (previo al proceso de laminación en caliente) por lo que no se consideran productos del proceso de fabricación de la placa. La identificación de la fase $\mathrm{U}(\mathrm{Al}, \mathrm{Si})_{3}$ con un parámetro de red de $a \sim 4.23 \AA$ (correspondiente a $17 \%$ de Si según [14]) es asociada a uno de los productos que forman la ZI. La baja intensidad relativa de sus reflexiones respecto de otras fases permite inferir que ésta es una fase minoritaria en la ZI. Además, un conjunto de reflexiones de alta intensidad es observado en ambos difractogramas. De todos los aluminuros y siliciuros de $\mathrm{U}$ o compuestos ternarios Al-Si-U conocidos, el espectro teórico del $\mathrm{U}_{3} \mathrm{Si}_{2}$ (tP10 en notación de Pearson, parámetros de red $a \sim 7.33 \AA$ y $c \sim 3.90 \AA$ [14]) con sus parámetros de red modificados $a_{\text {modif }} \sim 7.56 \AA$ y $c_{\text {modif }} \sim 4.03 \AA$ explicaría las reflexiones de este conjunto a partir de una única fase.

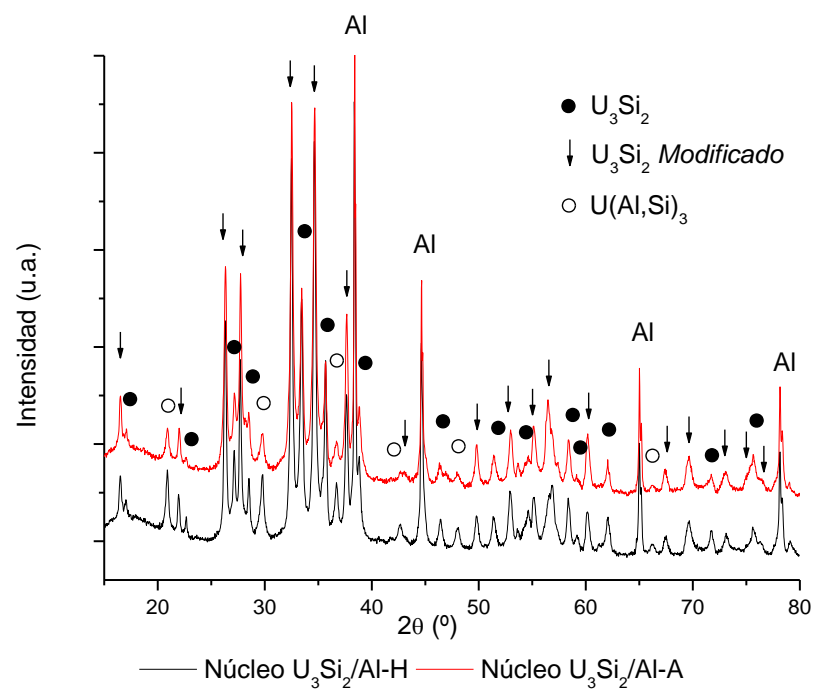

Figura 4: Muestras $\mathrm{U}_{3} \mathrm{Si}_{2} / \mathrm{Al}-\mathrm{H}$ y $\mathrm{U}_{3} \mathrm{Si}_{2} / \mathrm{Al}-\mathrm{A}$. Fases identificadas en el núcleo al finalizar el proceso de fabricación.

Las observaciones realizadas en alta magnificación en el modo de electrones retrodispersados revelaron, al menos, cuatro coloraciones claramente distinguibles las cuales estarían asociadas a las cuatro estructuras cristalinas diferentes presentadas en la Figura 4. Este modo de medición pone de manifiesto variaciones en la concentración siendo las zonas más oscuras más ricas en elementos de número atómico bajo mientras que en las más claras adquieren un mayor peso los elementos con número atómico más alto. Las Figuras 5, 6 y 7 muestran la distribución espacial y morfología de las cuatro fases presentes en los núcleos. De estas cuatro zonas, la de color negro corresponde a la matriz de Al puro mientras que las regiones blancas "1", las de color gris claro "2" y las de color gris oscuro "3" estarían asociadas a las partículas de siliciuro originales y a las dos fases formadas durante el proceso de fabricación. Es de destacar que para las zonas denominadas como 3 se identificaron dos morfologías diferentes: capa muy delgada $(<1 \mu \mathrm{m}$ de espesor) rodeando las partículas (Figura 5), o regiones de mayor tamaño ubicadas en la esquina de algunas partículas (Figura 6). 


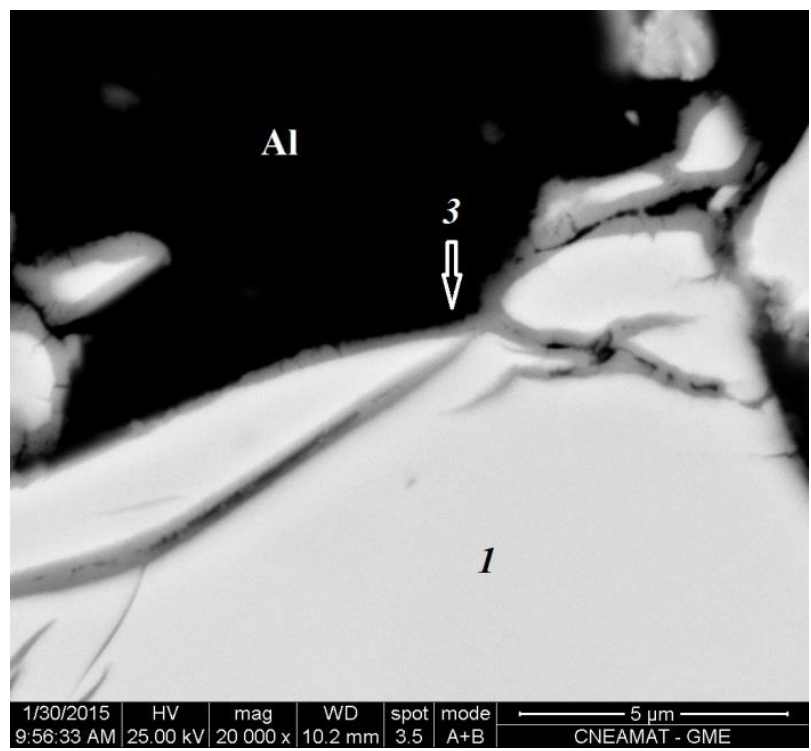

Figura 5: Muestra $\mathrm{U}_{3} \mathrm{Si}_{2} / \mathrm{Al}-\mathrm{H}$. Distribución espacial de las fases presentes en los núcleos: matriz de Al puro, Zona 1 y morfologia de banda delgada para la Zona 3.

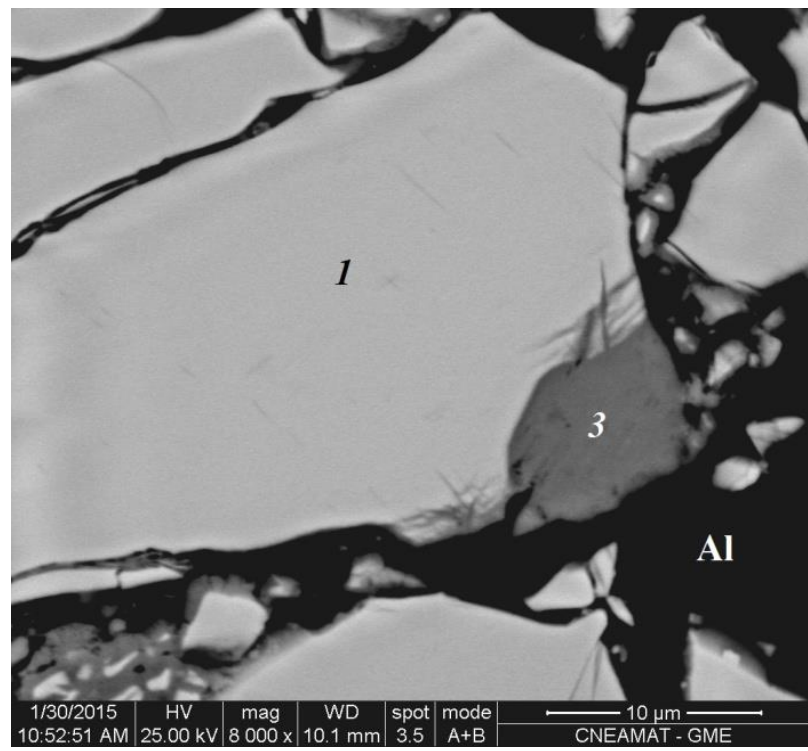

Figura 6: Muestra $\mathrm{U}_{3} \mathrm{Si}_{2} / \mathrm{Al}-\mathrm{H}$. Distribución espacial de las fases presentes en los núcleos: matriz de Al puro, Zona 1 y morfologia de regiones de mayor tamaño ubicadas en la esquina de algunas partículas para la Zona 3. 


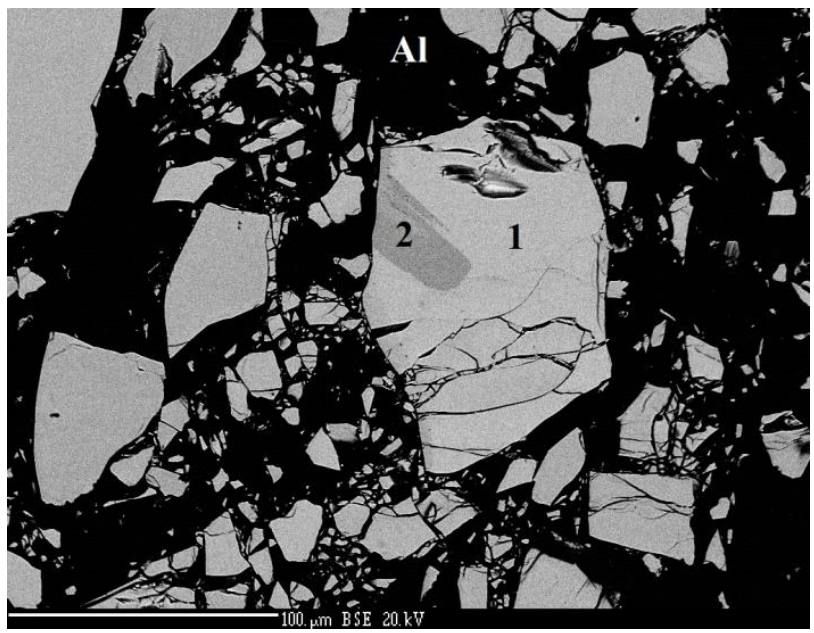

Figura 7: Muestra $\mathrm{U}_{3} \mathrm{Si}_{2} / \mathrm{Al}-\mathrm{H}$. Distribución espacial de las fases presentes en los núcleos: matriz de Al puro, Zona 1 y Zona 2.

Para determinar la concentración de las zonas $\mathbf{1}, 2$ y 3, y teniendo en cuenta la distribución, morfología y tamaño de cada una de ellas, se realizaron en algún caso una serie de mediciones puntuales convenientemente elegidas y/o un conjunto de barridos lineales, al azar, compuestos, como mínimo, por 30 mediciones puntuales cada uno. Los resultados de concentración obtenidos se resumen en la Tabla 2.

Tabla 2: Determinaciones de concentración de las zonas 1, 2 y 3 en las Figuras 5, 6 y 7. WDS.

\begin{tabular}{l|l|l|l|l}
\hline ZONA & COLOR EN FIGS. 5, 6 Y 7 & AL (\%AT) & SI (\%AT) & U (\%AT) \\
\hline $\mathbf{1}$ & Blanco & No detectado & $42.4 \pm 0.4$ & $57.6 \pm 0.4$ \\
\hline $\mathbf{2}$ & Gris claro & No detectado & $\sim 47$ & $\sim 53$ \\
\hline $\mathbf{3}$ & Gris oscuro & $\sim 56$ & $\sim 20$ & $\sim 24$ \\
\hline
\end{tabular}

De los resultados presentados en la Tabla 2, la identificación de estructuras cristalinas y las mediciones de concentración realizadas a las partículas de siliciuro luego del proceso de fundición (Tabla 1), se deduce que la Zona 1 corresponde a las partículas de $\mathrm{U}_{3} \mathrm{Si}_{2}$ de partida que no sufrieron ninguna modificación durante el proceso de fabricación. Para la Zona 3 las determinaciones de concentración mostraron una importante cantidad de $\mathrm{Al}$ y una relación $(\mathrm{Al}+\mathrm{Si}) / \mathrm{U} \sim 3$. Teniendo en cuenta la identificación de estructuras cristalinas, esta zona estaría asociada a la fase $\mathrm{U}(\mathrm{Al}, \mathrm{Si})_{3}$ pudiéndose observar además un buen acuerdo entre la concentración de Si obtenida por WDS, el parámetro de red, por XRD, y con la correlación parámetro de red vs \%Si propuesta en [14]. Por último, la Zona 2 estaría formada por la fase que se denominó $\mathrm{U}_{3} \mathrm{Si}_{2}$ modificada en la Figura 4 siendo su concentración más rica en $\mathrm{Si}$ y empobrecida en U respecto del valor obtenido para las partículas originales del compacto. Este enriquecimiento en Si (número atómico mucho menor que el del U) permite explicar que la Zona 2 tenga una coloración más oscura que la Zona 1 en la Figura 7. Dado que esta fase no fue identificada en la Figura 1, su formación es atribuida al proceso de fabricación de las placas.

\section{DISCUSIÓN}

En este trabajo se presenta el análisis de dos placas combustibles fabricadas con partículas de $\mathrm{U}_{3} \mathrm{Si}_{2}$ dispersadas en una matriz de $\mathrm{Al}$ puro. El objetivo perseguido con esta investigación es estudiar los compuestos formados por interdifusión entre el siliciuro de $\mathrm{U}$ y el Al durante el proceso de fabricación. Como es sabido, este tipo de combustibles se encuentran en la actualidad formando los núcleos de un gran número de reactores de investigación debido a su buen comportamiento bajo irradiación. En este sentido, resulta de gran importancia poder establecer semejanzas y diferencias entre este combustible y otros de mayor densidad, actualmente en desarrollo, como el U(Mo).

Existen en la literatura trabajos en donde los autores presentan resultados de estudios realizados sobre placas o miniplacas fabricadas con $\mathrm{U}_{3} \mathrm{Si}_{2}$ y matriz de $\mathrm{Al}$ puro $[15,16,17,18]$. Todos ellos concuerdan en la presencia de $\mathrm{U}(\mathrm{Al}, \mathrm{Si})_{3}$, dentro del núcleo, como única fase formada debido al proceso de fabricación. Al 
comparar esto respecto de los resultados obtenidos en nuestra investigación, existe acuerdo en la presencia de esta fase pero una diferencia respecto de la identificación de un segundo compuesto al que se denominó $\mathrm{U}_{3} \mathrm{Si}_{2}$ modificado por tener la misma estructura cristalina (tP10) que la fase $\mathrm{U}_{3} \mathrm{Si}_{2}$ pero con un aumento del volumen de celda del orden de $10 \%$ [14]. La intensidad de las reflexiones correspondientes a este compuesto estaría indicando una participación relativa importante dentro del compacto. Sin embargo, hasta el grado de avance de esta investigación, esto no presenta un buen acuerdo con las observaciones realizadas por SEM-BSE. En este sentido es necesario realizar una cuantificación de las fases identificadas por XRD y una nueva observación más detallada.

En el marco de la calificación de la aleación U(Mo) como combustible de alta densidad, se han realizado y publicado en todo el mundo una gran cantidad de investigaciones fuera de reactor ya sea mediante el estudio de pares de difusión o de placas (o miniplacas) luego del proceso de fabricación [11, 19, 20, 21, 22, 23]. A pesar de las diferencias en los distintos procesos termomecánicos utilizados, cuando $\mathrm{U}(\mathrm{Mo})$ es usado como aleación combustible y $\mathrm{Al}(\mathrm{Si})$ como matriz, todos los autores acuerdan en la formación de una zona de interacción formada por fases que contienen $\mathrm{Si}$ siendo los compuestos identificados: $\mathrm{U}(\mathrm{Al}, \mathrm{Si})_{3}, \mathrm{Si}_{2} \mathrm{U}, \mathrm{Si}_{5} \mathrm{U}_{3}$ y $\mathrm{Al}_{2} \mathrm{Si}_{3} \mathrm{U}_{3}$. Según las temperaturas y tiempos involucrados se observan diferencias respecto de la presencia y/o ausencia de alguno de ellos o la participación relativa dentro de la zona de interacción pero todos los autores reportan haber identificado $\mathrm{U}(\mathrm{Al}, \mathrm{Si})_{3}$ como el componente principal.

Como se expresara en el primer párrafo de la discusión, consideramos valiosa la comparación entre las reacciones generadas entre ambas aleaciones combustibles $\left(\mathrm{U}_{3} \mathrm{Si}_{2}\right.$ o $\left.\mathrm{U}(\mathrm{Mo})\right)$ y sus correspondientes matrices (Al puro o $\mathrm{Al}(\mathrm{Si})$ respectivamente) durante el proceso de fabricación. Focalizaremos esta comparación en miniplacas fabricadas bajo las mismas condiciones de modo que utilizaremos los resultados presentados en [11] y [23]. En estos trabajos se observó que cuando partículas de U(Mo) se encuentran inmersas en una matriz de $\mathrm{Al}-4 \%$ en peso $\mathrm{Si}$, se forma alrededor de todas las partículas de $\mathrm{U}(\mathrm{Mo})$ una zona de interacción de espesor uniforme $(\sim 1 \mu \mathrm{m})$ compuesta por $\mathrm{U}(\mathrm{Al}, \mathrm{Si})_{3}$ y $\mathrm{U}_{3} \mathrm{Si}_{5}$. Como puede observarse, el compuesto $\mathrm{U}(\mathrm{Al}, \mathrm{Si})_{3}$ se encuentra presente en las zonas de interacción de ambas aleaciones combustibles siendo la única diferencia la concentración de Si en el compuesto siendo sensiblemente mayor para miniplacas U(Mo)/Al(Si) que la obtenida en las placas $\mathrm{U}_{3} \mathrm{Si}_{2} / \mathrm{Al}$.

La formación de un mismo compuesto en las zonas de interacción de ambas aleaciones combustibles junto con el hecho de que dicho compuesto $\left(\mathrm{U}(\mathrm{Al}, \mathrm{Si})_{3}\right)$ no presenta problemas relacionados con su respuesta bajo irradiación, resulta auspicioso y permite suponer que una zona de interacción formada únicamente por este compuesto puede ser considerada "más apropiada" en el marco de la calificación de la aleación U(Mo).

\section{CONCLUSIONES}

En este trabajo, dos placas combustibles fabricadas con partículas de $\mathrm{U}_{3} \mathrm{Si}_{2}$ dispersadas en una matriz de $\mathrm{Al}$ puro fueron analizadas para identificar las fases presentes en la zona de interacción que se forma durante el proceso de fabricación. Los resultados se comparan con los obtenidos en miniplacas que contienen partículas atomizadas de U-7\% en peso Mo dispersas en una matriz de $\mathrm{Al}-4 \%$ en peso Si que experimentan el mismo proceso de fabricación. La única fase en común que se ha podido identificar es $\mathrm{U}(\mathrm{Al}, \mathrm{Si})_{3}$.

De acuerdo con los resultados presentados en este documento, y sabiendo que los combustibles fabricados a partir de siliciuros tienen un buen comportamiento bajo irradiación, se concluye que una zona de interacción compuesta únicamente por la fase $\mathrm{U}(\mathrm{Al}, \mathrm{Si})_{3}$ puede considerarse como "apropiada" de formarse durante el proceso de fabricación de combustibles $\mathrm{U}(\mathrm{Mo}) / \mathrm{Al}(\mathrm{Si})$ si se pretende que la misma actúe como capa protectora bajo irradiación.

En adelante, serán estudiados diversos pares de difusión $\mathrm{U}(\mathrm{Mo}) / \mathrm{Al}(\mathrm{Si})$ con el fin de establecer si existe una concentración "ideal" de Si (a incluir en la matriz de Al-Si) que pueda favorecer la formación de una zona de interacción monofásica compuesta por $\mathrm{U}(\mathrm{Al}, \mathrm{Si})_{3}$ en detrimento de la formación de otros compuestos U-Si.

\section{AGRADECIMIENTOS}

Los autores agradecen al personal técnico de la Gerencia Materiales y del Laboratorio de Difracción de Rayos X, Departamento Física de la Materia Condensada, Gerencia de Investigación y Aplicaciones, GAIyANN-CAC-CNEA por su colaboración en el desarrollo de este trabajo.

La Agencia Nacional de Promoción Científica y Tecnológica, a través del Fondo para la Investigación Científica y Tecnológica (FONCyT), ha financiado parcialmente este trabajo mediante el proyecto PICT- 
2010-1351 "Desarrollo de combustibles U(Mo)-Al(Si) utilizables en reactores nucleares de investigación y producción de radioisótopos para uso médico".

\section{BIBLIOGRAFÍA}

[1] MEYER, M.K., HOFMAN, G.L., HAYES, S.L., et al., "Low-temperature irradiation behavior of uranium-molybdenum alloy dispersion fuel", Journal of Nuclear Materials, v. 304, pp. 221-236, Ago. 2002.

[2] SNELGROVE, J., HOFMAN, G., MEYER, M., et al., "Development of very-high-density low-enricheduranium fuels", Nuclear Engeneering and Design, v. 178, pp. 119-126, Dic. 1997.

[3] LUNDBERG, L.B., "High-temperature interdiffusion and phase equilibria in U-Mo", Journal of Nuclear Materials, v. 167, pp. 64-75, Sept 1989.

[4] HOFMAN, G.L., FINLAY, M.R, KIM, Y.S., "Post-Irradiation Analysis of Low Enriched U-Mo/Al Dispersion Fuel Miniplate Tests, RERTR 4 and 5.", In: Proceedings of the 26th RERTR International Meeting, Viena, Austria, 2004.

[5] LEENAERS A., VAN DEN BERGHE, S., VAN EYKEN, J., et al., "Microstructural evolution of $\mathrm{U}(\mathrm{Mo})-\mathrm{Al}(\mathrm{Si})$ dispersion fuel under irradiation - Destructive analyses of the LEONIDAS E-FUTURE plates", Journal of Nuclear Materials, v. 441, pp. 439-448, Oct 2013.

[6] KEISER Jr, D.D., ROBINSON, A.B., JUE, J.F., et al., "Microstructural development in irradiated U7Mo/6061 Al alloy matrix dispersion fuel", Journal of Nuclear Materials, v. 393, pp. 311-329, Sep. 2009.

[7] KEISER Jr, D.D., JUE, J.F., WOOLSTENHULME, N.E., et al., "Potential annealing treatments for tailoring the starting microstructure of low-enriched U-Mo dispersion fuels to optimize performance during irradiation", Journal of Nuclear Materials, v. 419, pp. 226-234, Sep 2011.

[8] KOONEN, E., GUYON, H., LEMOINE, P., et al., "The LEONIDAS and US Partnership for UMo Dispersion Fuel Qualification", In: Proceedings of the 31th RERTR International Meeting, Beijing, China, Nov. 2009.

[9] FRÉRY, F., GUYON, H., KOONEN, E., et al., "LEONIDAS UMo Dispersion Fuel Qualification Program: Progress and Prospects", In: Proceedings of the 32nd RERTR International Meeting, Lisboa, Portugal, Oct. 2010.

[10] CHAROllais, F., LeMOINE, P., CAlZAVARA, Y., et al., "LEONIDAS U(Mo) Dispersion Fuel Qualification Program: Progress and Perspectives", In: Proceedings of the 33rd RERTR International Meeting, Santiago, Chile, Oct. 2011.

[11] MIRANDOU, M., ARICÓ, S., BALART, S., et al., "Development of U(Mo) dispersión fuel miniplates with $\mathrm{Al}(\mathrm{Si})$ matrix. Microstructural characterization." In: Proceedings of the International Meeting on Research Reactors Fuel Management (RRFM), pp. 134-147, Abr 2014.

[12] ARICÓ, S., MIRANDOU, M., BALART, S., et al., "Desarrollo de miniplacas combustibles dispersas de $\mathrm{U}(\mathrm{Mo})$ en matriz $\mathrm{Al}(\mathrm{Si})$. Caracterización microstructural de núcleos", In: Memorias del $13^{\circ}$ Congreso Internacional en Ciencia y Tecnología de Metalurgia y Materiales SAM-CONAMET, Misiones, Argentina, Ago 2013.

[13] KRAUS, W., NOLZE, G., MÜLLER, U., "Pulverdiffraktogramme aus Einkristalldaten und Anpassung experimenteller Beugungsaufnahmen", PowderCell 2.3., Mar 2000.

[14] DWIGHT, A., "A study of the uranium aluminum silicon system", Report Specification, ANL 82-14, pp. 1-39, Sep. 1982.

[15] NAZARE, S., "The status of development of LEU-fuels for research reactors", Materials Science Forum, v. 48-49, pp. 297-314, Mar 1989.

[16] LEENAERS, A., VAN DEN BERGHE, S., KOONEN, E., et al., Microstructure of $\mathrm{U}_{3} \mathrm{Si}_{2}$ fuel plates submitted to a high heat flux, Journal of Nuclear Materials, v. pp. 327 121-129, Ene. 2004.

[17] MARÍN, J., LISBOA, J., URETA, J., et al., "Synthesis and clad interaction study of $\mathrm{U}_{3} \mathrm{Si}_{2}$ powders dispersed in an aluminum matrix", Journal of Nuclear Materials, v. 228, pp. 61-67, Sept. 1996.

[18] KIM, K-H., PARK, J-M, KIM, C-K., et al., "Thermal compatibility studies of $\mathrm{U}_{3} \mathrm{Si}_{2}$ dispersion fuels prepared with centrifugally atomized powder", Journal of Nuclear Materials, v. 270, pp 315-321, Dic. 1998.

[19] KEISER Jr, D.D., JUE, J.F., YAO, B., et al., "Microstructural characterization of U-7Mo/Al-Si alloy matrix dispersion fuel plates fabricated at 500 ${ }^{\circ}$ ", Journal of Nuclear Materials, v. 412 pp. 90-99, Feb 2011. 
[20] JUNGWIRTH, R., PALANCHER, H., BONNIN, A., et al., "Microstructure of as-fabricated $\mathrm{UMo} / \mathrm{Al}(\mathrm{Si})$ plates prepared with ground and atomized powder", Journal of Nuclear Materials, v. 438 pp. 246-260, Mar 2013.

[21] YAO, B., PEREZ, E., KEISER Jr., D.D., et al., "Microstructure characterization of as-fabricated and $475{ }^{\circ} \mathrm{C}$ annealed U-7 wt.\% Mo dispersion fuel in Al-Si alloy matrix", Journal of Alloys and Compounds, v. 509, pp. 9487-9496, Jul 2011.

[22] PALANCHER, H., BONNIN, A., HONKIMÄKI, V., et al., "Quantitative crystallographic analysis of as-fabricated full size U-Mo/Al(Si) nuclear fuel plates", Journal of Alloys and Compounds, v. 527, pp. 53-65, Feb 2012.

[23] MIRANDOU, M., ARICÓ, S., BALART, S., et al., "Comparison between U(Mo)/Al(Si) miniplate and $\mathrm{U}_{3} \mathrm{Si}_{2} / \mathrm{Al}$ miniplate after the same fabrication process", In: Proceedings of the International Meeting on Research Reactors Fuel Management (RRFM), pp. 798-806, Bucharest, Romania, Abr. 2015. 